\title{
THE MAZUR PROPERTY AND COMPLETENESS IN THE SPACE OF BOCHNER-INTEGRABLE FUNCTIONS $L_{1}(\mu, X)$
}

\author{
by G. SCHLÜCHTERMANN
}

(Received 30 January, 1991)

1. Introduction and preliminaries. A locally convex space $(E, \mathscr{T})$ has the Mazur Property if and only if every linear $\mathscr{T}$-sequential continuous functional is $\mathscr{T}$-continuous (see [11]).

In the Banach space setting, a Banach space $X$ is a Mazur space if and only if the dual space $X^{*}$ endowed with the $w^{*}$-topology has the Mazur property. The Mazur property was introduced by S. Mazur, and, for Banach spaces, it is investigated in detail in [4], where relations with other properties and applications to measure theory are listed. T. Kappeler obtained (see [8]) certain results for the injective tensor product and showed that $L_{1}(\mu, X)$, the space of Bochner-integrable functions over a finite and positive measure space $(S, \Sigma, \mu)$, is a Mazur space provided $X$ is also, and $\ell_{1}$ does not embed in $X$.

The same result is established, using a different technique, in the second section. It can be shown that this result can be extended: for every separable measure space, $L_{1}(\mu, X)$ enjoys the Mazur property if and only if $X$ does. Since D. Leung has already obtained this result independently (see $[9,3.1$. Theorem]), it is only stated without proof. The deductions here are obtained by considering the dual pairing $\left\langle L_{1}(\mu, X), L_{\infty}\left(\mu, X^{*}\right)\right\rangle$, where $L_{\infty}\left(\mu, X^{*}\right)$ is the space of $X^{*}$-valued, $\mu$-essentially bounded functions. If $\sigma^{\prime}$ and $\sigma^{*}$ denote the weak topologies of this pairing on $L_{1}(\mu, X)$ and $L_{\infty}\left(\mu, X^{*}\right)$, respectively, then the locally convex space $\left(L_{1}(\mu, X), \sigma^{\prime}\right)$ has the Mazur property and $\left(L_{\infty}\left(\mu, X^{*}\right), \sigma^{*}\right)$ has the Mazur property if and only if $\left(X^{*}, w^{*}\right)$ has the Mazur property. The fact that $\left(L_{1}(\mu, X), \sigma^{\prime}\right)$ has the Mazur property will establish a simple alternative proof of a result of N. Ghoussoub and P. Saab (see [6, Theorem 1]).

The third section will show that the locally convex spaces $\left(L_{1}(\mu, X), \tau^{\prime}\right)$, $\left(L_{\infty}\left(\mu, X^{*}\right), \tau^{*}\right)$ are complete, where $\tau^{\prime}, \tau^{*}$ denote the Mackey-topologies of the dual pairing $\left\langle L_{1}(\mu, X), L_{\infty}\left(\mu, X^{*}\right)\right\rangle$ on $L_{1}(\mu, X), L_{\infty}\left(\mu, X^{*}\right)$, respectively. This extends a result of $\mathrm{T}$. Kappeler who proved that $\left(L_{1}(\mu, X), \tau^{\prime}\right)$ is complete provided that $L_{1}(\mu, X)$ is a Mazur space.

Throughout the paper, $B(Y)$ denotes the unit ball of a Banach space $Y$, while $\operatorname{co}(A)$ and $\operatorname{aco}(A)$ are the convex hull and absolutely convex hull, respectively, of a subset $A \subset Y$ of a Banach space $Y$. For a finite measure space $(S, \Sigma, \mu), \pi$ is a finite $\Sigma$-partition and

$$
\Pi=\left\{\left(\pi_{n}\right)_{n \in \mathbb{N}} ;\left(\pi_{n}\right)_{n \in \mathbb{N}} \text { is an increasing sequence of finite partitions }\right\} .
$$

For a finite partition $\pi$, let $\mathbf{E}_{\pi}$ be the conditional expectation operator on the $\sigma$-algebra generated by $\pi$. Define

$$
V_{\infty}\left(\mu, X^{*}\right)=\left\{m: \Sigma \rightarrow X^{*}, \exists K>0, \forall A \in \Sigma,\|m(A)\| \leq K \mu(A)\right\},
$$

the Banach space of $\mu$-Lipschitz continuous $X^{*}$-valued vector measures equipped with the norm $\|m\|_{\text {Lip }}=\inf \{K>0, \forall A \in \Sigma,\|m(A)\| \leq K \mu(A)\}$. Note that $V_{\infty}\left(\mu, X^{*}\right)$ is isometrically isomorphic to the dual $L_{1}(\mu, X)^{*}$ (see [2]). All these results exploit the following 
characterization of certain $X$ or $X^{*}$ valued vector measures which was first given in this form by J. Batt and W. Hiermeyer (see [1, 2.4. Proposition]).

If $\left(\pi_{n}\right)_{n \in \mathbb{N}} \in \Pi$ then set

$$
\begin{aligned}
& \Lambda_{1}\left(\left(\pi_{n}\right)_{n \in \mathbb{N}}\right)=\left\{T: L_{1}(\mu, X) \rightarrow c_{0} ; \exists\left(g_{n}\right)_{n \in \mathbb{N}} \subset B\left(L_{\infty}\left(\mu, X^{*}\right)\right)\right. \\
&\text { such that } \left.g_{n}=\left(\mathbf{E}_{\pi_{n+1}}-\mathbf{E}_{\pi_{n}}\right)\left(g_{n}\right) \text { and } T f=\left(\left\langle f, g_{n}\right\rangle\right)_{n \in \mathbb{N}}\right\}, \\
& \Lambda_{1}^{*}\left(\left(\pi_{n}\right)_{n \in \mathbb{N}}\right)=\left\{T: L_{\infty}\left(\mu, X^{*}\right) \rightarrow c_{0} ;\right. \exists\left(f_{n}\right)_{n \in \mathbb{N}} \subset B\left(L_{\infty}(\mu, X)\right) \\
&\text { such that } \left.f_{n}=\left(\mathbf{E}_{\pi_{n+1}}-\mathbf{E}_{\pi_{n}}\right)\left(f_{n}\right) \text { and } T g=\left(\left\langle f_{n}, g\right\rangle\right)_{n \in \mathbb{N}}\right\} .
\end{aligned}
$$

These classes of operators help to characterize vector measures which have a Bochner density as is shown in the next lemmata; the proofs are basically the same as in $[1,2.4$. Proposition]).

1.1. LeMmA. Let $m: \Sigma \rightarrow X$ be a finitely additive vector measure of bounded variation such that $\mu(A)=0$ implies $m(A)=0$. Then the following are equivalent:

(a) $m$ has a $L_{1}(\mu, X)$-density; where

(b) $\forall\left(\pi_{n}\right)_{n \in \mathbb{N}} \in \Pi, \forall T \in \Lambda_{1}\left(\left(\pi_{n}\right)_{n \in \mathbb{N}}\right), T\left(K\left(\left(\pi_{n}\right)_{n \in \mathbb{N}}\right)\right)$ is relatively weakly compact,

$$
K\left(\left(\pi_{n}\right)_{n \in \mathbb{N}}\right)=\left\{\sum_{A \in \pi_{n}} \frac{m(A)}{\mu(A)} \chi_{A} ; n \in \mathbb{N}\right\} .
$$

1.2. Lemma. For $m \in V_{\infty}\left(\mu, X^{*}\right)$ the following are equivalent:

(a) $m$ has a Bochner-density $g \in L_{\infty}\left(\mu, X^{*}\right)$;

(b) $\forall\left(\pi_{n}\right)_{n \in \mathbb{N}} \in \Pi, \forall T \in \Lambda_{1}^{*}\left(\left(\pi_{n}\right)_{n \in \mathbb{N}}\right), T\left(K\left(\left(\pi_{n}\right)_{n \in \mathbb{N}}\right)\right)$ is relatively weakly compact, where

$$
K\left(\left(\pi_{n}\right)_{n \in \mathbb{N}}\right)=\left\{\sum_{A \in \pi_{n}} \frac{m(A)}{\mu(A)} \chi_{A} ; n \in \mathbb{N}\right\}
$$

2. The Mazur property for $L_{1}(\mu, X)$.

2.1. Theorem. $\left(L_{1}(\mu, X), \sigma^{\prime}\right)$ has the Mazur property.

Proof. Let $G \in L_{1}(\mu, X)^{*}$ be $\sigma^{\prime}$-sequential continuous. The representation of the dual $L_{1}(\mu, X)^{*}$ shows (see the introduction)

$$
\exists m \in V_{\infty}\left(\mu, X^{*}\right) \text {, with }\langle f, G\rangle=\int f d m \text { as the Bartle-integral. }
$$

Hence the proof is done if $m$ has a Bochner-density. Thus, applying (1.2.), let $\left(\pi_{n}\right)_{n \in \mathbb{N}} \in \Pi$ and $T \in \Lambda_{1}^{*}\left(\left(\pi_{n}\right)_{n \in \mathbb{N}}\right)$. Define, for $k \in \mathbb{N}$,

$$
h_{k}=\sum_{A \in \pi_{k}} \frac{m(A)}{\mu(A)} \chi_{A} .
$$

If $\left(f_{n}\right)_{n \in \mathbb{N}}$ is the martingale difference sequence related to $T$ then it is easy to realize that $\left(f_{n}\right)$ converges to 0 in $\left(L_{1}(\mu, X), \sigma^{\prime}\right)$. To demonstrate that $\left(T\left(h_{k}\right)\right)_{n \in \mathbb{N}}$ is relatively weakly 
compact one proves that

$$
T\left(h_{k}\right) \rightarrow\left(\left\langle f_{n}, h_{n+1}\right\rangle\right)_{n \in \mathbb{N}} \text { weakly in } c_{0} \text { if } k \rightarrow \infty .
$$

To see this, note that,

$$
\forall n \in \mathbb{N}, \quad\left\langle f_{n}, G\right\rangle=\left\langle f_{n}, \mathbf{E}_{\pi_{n+1}}^{*} G\right\rangle=\left\langle f_{n}, h_{n+1}\right\rangle .
$$

Since $\left(f_{n}\right)$ is $\sigma^{\prime}$-convergent to 0 , the $\sigma^{\prime}$-sequential continuity of $G$ implies

$$
\left\langle f_{n}, G\right\rangle \rightarrow 0 \text { if } n \rightarrow \infty .
$$

Since $f_{n}=\left(\mathbf{E}_{\pi_{n+1}}-\mathbf{E}_{\pi_{n}}\right)\left(f_{n}\right)$, it follows that

$$
T\left(h_{k}\right) \rightarrow\left(\left\langle f_{n}, h_{n+1}\right\rangle\right)_{n \in \mathbb{N}} \text { pointwise if } k \rightarrow \infty .
$$

Thus, by (2), (3) and the boundedness of $\left(h_{k}\right)_{k \in \mathbb{N}} \subset L_{\infty}\left(\mu, X^{*}\right)$, the sequence $\left(T\left(h_{k}\right)\right)_{k \in \mathbb{N}}$ converges weakly and (1) is proved. Hence there is a $g \in L_{\infty}\left(\mu, X^{*}\right)$ such that, for all $f \in L_{1}(\mu, X)$,

$$
\langle f, g\rangle=\langle f, G\rangle,
$$

and the proof is finished.

The next two corollaries will deal with weakly compact and $\sigma^{\prime}$-compact subsets of $L_{1}(\mu, X)$ and are applications of (2.1).

2.2. Corollary. Every relatively $\sigma^{\prime}$-compact subset of $L_{1}(\mu, X)$ is relatively weakly compact if and only if $X^{*}$ has the Radon-Nikodym property with respect to $(S, \Sigma, \mu)$.

2.3. Corollary (N. Ghoussoub, P. Saab). All bounded and uniformly integrable subsets $K \subset L_{1}(\mu, X)$ such that $\left\{\int_{A} f d \mu ; f \in K\right\}$ is relatively weakly compact in $X$ for all $A \in \Sigma$ are relatively weakly compact in $L_{1}(\mu, X)$ if and only if both $X$ and $X^{*}$ have the Radon-Nikodym property with respect to $(S, \Sigma, \mu)$.

The technique used in (2.1) applied in the dual version to $L_{\infty}\left(\mu, X^{*}\right)$ leads to the following theorem, of which part (b) is stated independently in $[9,3.1]$.

2.4. THEOREM. (a) $\left(L_{\infty}\left(\mu, X^{*}\right), \sigma^{*}\right)$ has the Mazur property if and only if $\left(X^{*}, w^{*}\right)$ has the Mazur property.

(b) If $L_{\infty}\left(\mu, X^{*}\right)$, is $w^{*}$-sequentially dense in $L_{1}\left(\mu, X^{*}\right)$ then $L_{1}(\mu, X)$ is a Mazur space if and only if $X$ is.

In particular if either the measure space $(S, \Sigma, \mu)$ is separable or $\ell_{1}$ does not embed in $X$ then $L_{1}(\mu, X)$ is a Mazur space if and only if $X$ is.

3. Completeness in the dual pairing $\left\langle L_{1}(\mu, X), L_{\infty}\left(\mu, X^{*}\right)\right\rangle$. The space $\left(L_{1}(\mu, X), \sigma^{\prime}\right)$ is, due to an unpublished result of $J$. Batt and W. Hiermeyer, sequentially complete if and only if $X$ is weakly sequentially complete and $X$ has the Radon-Nikodym property with respect to $(S, \Sigma, \mu)$ (see also [10,3.3. Lemma]). By [3, IV.1, Theorem 1] it is evident that $\left(L_{\infty}\left(\mu, X^{*}\right), \sigma^{*}\right)$ is sequentially complete if and only if $X^{*}$ has the Radon-Nikodym property with respect to $(S, \Sigma, \mu)$. The unknown fact if the corresponding Mackey topologies are complete was partially solved by $T$. Kappeler (see [8, 4.2. Proposition]). The next theorem will show that they both are complete locally convex spaces. 
3.1. TheOrem. The spaces $\left(L_{1}(\mu, X), \tau^{\prime}\right)$ and $\left(L_{\infty}\left(\mu, X^{*}\right), \tau^{*}\right)$ are complete.

Proof. Let $\left(f_{\alpha}\right)_{\alpha \in J}$ be a $\tau^{\prime}$-Cauchy net in $L_{1}(\mu, X)$.

Step 1. Since the operator

$$
\begin{aligned}
I_{A}: L_{1}(\mu, X) & \rightarrow X \\
f & \mapsto \int_{A} f d \mu
\end{aligned}
$$

is $\tau^{\prime}-\|\cdot\|$-continuous, a finitely additive vector measure can be defined as follows:

$$
\begin{aligned}
m: \Sigma & \rightarrow X \\
A & \mapsto\|\cdot\| \lim _{\alpha \in J} I_{A}\left(f_{\alpha}\right) .
\end{aligned}
$$

It is easy to check that $\mu(A)=0$ implies $m(A)=0$. To apply (1.1), $m$ has to have bounded variation. To see this, consider $L_{1}(\mu, X)$ as a subspace of $L_{\infty}\left(\mu, X^{*}\right)^{*}$; then the net $\left(f_{\alpha}\right)$ is a Cauchy-net in the dual Mackey topology $\tau\left(L_{\infty}\left(\mu, X^{*}\right)^{*}, L_{\infty}\left(\mu, X^{*}\right)\right)$. Since the dual Mackey topology is complete (see [11, 9-2-14 Theorem]), this net has a limit $F \in$ $L_{\infty}\left(\mu, X^{*}\right)^{*}$. In particular

$$
\left\langle f_{\alpha}, g\right\rangle \rightarrow\langle F, g\rangle \forall g \in L_{\infty}\left(\mu, X^{*}\right) \quad \text { if } \quad \alpha \in J
$$

Since $F \in L_{\infty}\left(\mu, X^{*}\right)^{*}$, a finitely additive vector measure of bounded variation can be defined in the following way:

$$
\begin{aligned}
m^{\prime}: \Sigma & \rightarrow X^{* *} \\
A & \mapsto\left(x^{*} \mapsto\left\langle F, x^{*} \chi_{A}\right\rangle\right) .
\end{aligned}
$$

Evidently $m$ and $m^{\prime}$ coincide by the definition of $m$ and (4). Hence

$$
\langle F, g\rangle=\int_{S} g d m \text { for all simple functions } g=\sum_{i=1}^{n} x_{i}^{*} \chi_{A_{i}} \text { in } L_{\infty}\left(\mu, X^{*}\right) .
$$

Since $\left(f_{\alpha}\right)$ converges in the dual Mackey topology to $F \in L_{\infty}\left(\mu, X^{*}\right)^{*}$, it follows that

$$
\langle F, g\rangle=\int_{S} g d m \forall g \in L_{\infty}\left(\mu, X^{*}\right) .
$$

Step 2: $m$ has a Bochner derivative. Use (1.1) and select a $\left(\pi_{n}\right)_{n \in \mathbb{N}} \in \Pi$ and $T \in \Lambda_{1}\left(\left(\pi_{n}\right)_{n \in \mathbb{N}}\right)$. Define, for $n \in \mathbb{N}$,

$$
h_{n}=\sum_{A \in \pi_{n}} \frac{m(A)}{\mu(A)} \chi_{A} .
$$

Let $\left(g_{n}\right)_{n \in \mathbb{N}} \subset B\left(L_{\infty}\left(\mu, X^{*}\right)\right)$ be the martingale difference sequence related to $T$. Then $\left(g_{n}\right)$ converges to 0 in $\left(L_{\infty}\left(\mu, X^{*}\right), \sigma^{*}\right)$. With the help of (1.2), it can be shown along the line of the proof of $\left[1,2.5\right.$. Theorem] that $\overline{\mathrm{aco}} \sigma^{*}\left(g_{n} ; n \in \mathbb{N}\right)$ is $\sigma^{*}$-compact. Hence the set

$$
D=\left\{\sum_{n=1}^{\infty} b_{n} g_{n} ;\left(b_{n}\right)_{n \in \mathbb{N}} \subset B\left(\ell_{1}\right)\right\}
$$

is $\sigma^{*}$-compact. 
Suppose now that $\left(T\left(h_{n}\right)\right)_{n \in \mathbb{N}}$ is not relatively weakly compact in $c_{0}$. According to [7, Theorem 1],

$\exists \theta>0, \exists\left(h_{n_{k}}\right)_{k \in \mathbb{N}}$, subsequence $\exists\left(b^{\prime}\right)_{l \in \mathbb{N}} \in B\left(\ell_{1}\right)$ such that

$$
\inf \left\{\left\langle T\left(h_{n_{k}}\right), b^{\prime}\right\rangle ; l<k\right\}>\sup \left\{\left\langle T\left(h_{n_{k}}\right), b^{\prime}\right\rangle ; l>k\right\}+\theta .
$$

Since $\left(f_{\alpha}\right)$ is a $\tau^{\prime}$-Cauchy net and $D$ is $\sigma^{*}$-compact,

$$
\exists \alpha_{0} \in J, \forall \alpha, \beta \geq \alpha_{0}, \quad \sup _{g \in D}\left|\left\langle f_{\alpha}-f_{\beta}, g\right\rangle\right|<\frac{\theta}{8} .
$$

The definition of the vector measure $m$ reveals

$$
\forall k \in \mathbb{N}, \exists \alpha(k) \geq \alpha_{0}, \quad\left\|h_{n_{k}}-\mathbf{E}_{\pi_{n_{k}}}\left(f_{\alpha(k)}\right)\right\|<\frac{\theta}{8} .
$$

The martingale $\left(\mathbf{E}_{\pi_{n_{k}}}\left(f_{\alpha_{0}}\right) ; k \in \mathbb{N}\right)$ is relatively weakly compact since it converges in norm. Thus, by [7, Theorem 1],

$$
\inf \left\{\left\langle T\left(\mathbf{E}_{\pi_{n_{k}}}\left(f_{\alpha_{0}}\right)\right), b^{\prime}\right\rangle ; l<k\right\} \leq \sup \left\{\left\langle T\left(\mathbf{E}_{\pi_{n_{k}}}\left(f_{\alpha_{0}}\right)\right), b^{\prime}\right\rangle ; l>k\right\} .
$$

Finally, using all the above facts, one computes for $k, l \in \mathbb{N}$ :

$$
\begin{aligned}
\left|\left\langle T\left(h_{n_{k}}\right)-T\left(\mathbf{E}_{\pi_{n_{k}}}\left(f_{\alpha_{0}}\right)\right), b^{\prime}\right\rangle\right|= & \left|\left\langle h_{n_{k}}-\mathbf{E}_{\pi_{n_{k}}}\left(f_{\alpha_{0}}\right), \sum_{j=1}^{\infty} b_{j}^{\prime} g_{j}\right\rangle\right| \\
\leq & \left|\left\langle h_{n_{k}}-\mathbf{E}_{\pi_{n_{k}}}\left(f_{\alpha(k)}\right), \sum_{j=1}^{\infty} b_{j}^{\prime} g_{j}\right\rangle\right| \\
& +\left|\left\langle\mathbf{E}_{\pi_{n_{k}}}\left(f_{\alpha(k)}\right)-\mathbf{E}_{\pi_{n_{k}}}\left(f_{\alpha_{0}}\right), \sum_{j=1}^{\infty} b_{j}^{\prime} g_{j}\right\rangle\right| \\
& <\frac{\theta}{8}+\left|\left\langle f_{\alpha(k)}-f_{\alpha_{0}}, \sum_{j=1}^{n_{k}-1} b_{j}^{\prime} g_{j}\right\rangle\right| \quad \text { (by (8)) } \\
& <\frac{\theta}{8}+\frac{\theta}{8} \text { (according to (7)) } \\
= & \frac{\theta}{4} .
\end{aligned}
$$

By consideration of (6), this forms a contradiction to (9), and hence $m$ has a derivative $f \in L_{1}(\mu, X)$.

Since $\left(L_{1}(\mu, X), \tau^{\prime}\right)$ has a zero neighborhood basis of $\sigma^{\prime}$-closed sets, we have

$$
f_{\alpha} \rightarrow f \text { for } \alpha \in J \text { in } \tau^{\prime} \text { if and only if } f_{\alpha} \rightarrow f \text { for } \alpha \in J \text { in } \sigma^{\prime} .
$$

Hence the completeness of $\left(L_{1}(\mu, X), \tau^{\prime}\right)$ follows from (5) since,

$$
\forall g \in L_{\infty}\left(\mu, X^{*}\right), \quad \lim _{\alpha \in J}\left\langle f_{\alpha}, g\right\rangle=\langle F, g\rangle=\langle f, g\rangle .
$$

Since $\left(L_{1}(\mu, X), \tau^{\prime}\right)$ is complete as shown above, $\left(L_{1}(\mu, X), \sigma^{\prime}\right)$ has the convex compactness property. Hence by $[11,9-2-4$ Theorem $]$, the space $\left(L_{\infty}\left(\mu, X^{*}\right), \tau^{*}\right)$ is also complete. 
The following two corollaries can be obtained from the above theorem and abstract results in locally convex theory. The first one was already observed by J. Batt and W. Hiermeyer (see [1, 2.6. Corollary]). The second one is a James type result (see e.g. [5]).

3.2. Corollary. The convex (or absolutely convex) hull of a relatively $\sigma^{\prime}$-compact subset is again relatively $\sigma^{\prime}$-compact.

3.3. Corollary. A $\sigma^{\prime}$-closed and bounded subset $K \subset L_{1}(\mu, X)$ is $\sigma^{\prime}$-compact if and only if every $g \in L_{\infty}\left(\mu, X^{*}\right)$ attains its suprema on $K$.

\section{REFERENCES}

1. J. Batt and W. Hiermeyer, On compactness in $L_{p}(\mu, X)$ in the weak topology and in the topology $\sigma\left(L_{p}(\mu, X), L_{q}\left(\mu, X^{\prime}\right)\right)$, Math. $Z .182$ (1983), 409-432.

2. J. Diestel, Remarks on weak compactness in $L_{1}(\mu, X)$, Glasgow Math. J. 18 (1977), 87-91.

3. J. Diestel and J. J. Uhl, Jr., Vector measures, Mathematical Surveys No. 15 (American Mathematical Society, 1977).

4. G. Edgar, Measurability in a Banach space, II, Indiana Univ. Math. J. 28 (1979), 559-579.

5. K. Floret, Weakly compact sets, Lecture Notes in Mathematics 801 (Springer, 1980).

6. $N$. Ghoussoub and $P$. Saab, Weak compactness in spaces of Bochner integrable functions and the Radon-Nikodym property, Pacific J. Math. 110 (1984), 65-70.

7. R. C. James, Weak compactness and reflexivity, Israel J. Math. 2 (1964), 101-119.

8. T. Kappeler, Banach spaces with the condition of Mazur, Math. Z. 191 (1981), 623-631. $51-54$.

9. D. H. Leung, On Banach Spaces with Mazur's property, Glasgow Math. J. 33 (1991),

10. G. Schlüchtermann and R. F. Wheeler, On strongly WCG Banach spaces, Math. Z. 199 (1988), 387-398.

11. A. Wilansky, Modern methods in topological vector spaces (McGraw-Hill, 1978).

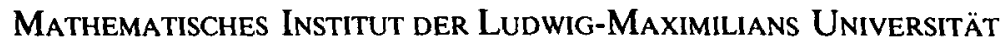

ThERESIENSTRASSE 39

W-8000 MÜNCHEN 2

Germany 